\title{
Special issue on optimization and variational analysis, dedicated to Professor Marco Antonio López Cerdá on the occasion of his 60th birthday
}

\author{
María Josefa Cánovas · Juan Parra
}

Published online: 8 June 2012

(C) Sociedad de Estadística e Investigación Operativa 2012

This special issue arose in relation to the 4th Workshop on Optimization and Variational Analysis, in honor of Professor Marco A. López on his 60th birthday (http://icio.umh.es/congresos/ova4mlopez/), held in Elche (Alicante, Spain) on 1416 June 2010, with 31 invited speakers and around 70 participants. The workshop was organized by the Optimization and Stability research group at the Miguel Hernández University of Elche (M.J. Cánovas, F.J. Gómez-Senent, J. Parra, and F.J. Toledo), with the valuable help of A. Meca. We all would like to thank the Editors-in-chief of TOP, Profs. Carrizosa and Puerto for their invitation to promote this issue, as well as all authors and discussants for their valuable contributions.

We are aware that the enthusiastic response to our call, for both the workshop and the present special issue, obeys the magnetism of the figure of Marco in his scientific and human components. With his versatile personality (highly reputed mathematician, excellent teacher, great friend, friendly person, etc.), he has promoted many scientific careers in Spain. As a part of this privileged group of people who were (and are forever) his disciples, we would like to show from these pages our deepest gratitude to him. Congratulations, Marco!

A brief biography Marco Antonio López Cerdá was born on 1 August 1949 in Alcoy, in the province of Alicante, Spain. He graduated in Mathematics in 1971 at Valencia University, where he defended his Ph.D. thesis in 1973. In 1981 he became a full professor at Valencia University and in 1985 moved to Alicante University, where he is a professor on statistics and operations research till the present.

Marco López has been a teacher for several generations of Spanish mathematicians and adviser or coadviser of $17 \mathrm{Ph} . \mathrm{D}$. students in several areas such as semi-infinite

M.J. Cánovas · J. Parra ( $₫)$

Miguel Hernández University, Elche, Spain

e-mail: parra@umh.es 
programming, network flows, assignment problem, convex optimization, graphs, scheduling, game theory, and variational analysis. He has been a leader of several projects, both theoretical (basic research) and applied (for the university community services, the city and the province of Alicante, and bank branches networks).

His 1998 book (with M.A. Goberna) on Linear Semi-Infinite Optimization has become a classical in the field. His activity level is increasing with the time: half of his more than 100 papers in refereed journals have been published in the last decade.

The cooperative aspect of Marco López and his service to the mathematical community is also remarkable. He has been an organizer or coorganizer of several important national and international meetings. We underline the International Workshop on Semi-Infinite Programming and Related Topics (Alicante 1999), the International Workshop on Semi-Infinite Programming, (Tainan, Taiwan, 2005), the Iberian Conference in Optimization (Coimbra, Portugal, 2006), and the 11th International Workshop on Well-Posedness of Optimization Problems and Related Topics (Alicante 2007). He has also been the chair of the program committee of the EUROPT workshops in Remagen (Germany) 2009 and Aveiro (Portugal) 2010, as satellite meetings of the EURO conference, organized by the EURO working group in continuous optimization.

Other positions related to his service to the scientific community are: a coeditor in chief of TOP from 2000 to 2007, the elected vice-president of SEIO (the Spanish society of Statistics and Operations Research) in 1985 and 1998, the elected president of SEIO in 1986, the chair of EUROPT since 2008 to 2010, and a member of the directors board of i-MATH Consolider, 2006-2011, a huge project of the Spanish Ministry of Education for all areas of mathematics, gathering more than 300 research groups, and with a budget of 7.5 million euros for the whole period of five years. Marco López has been the main coordinator of this project since July 2008 until the end of the project in April 2012. 